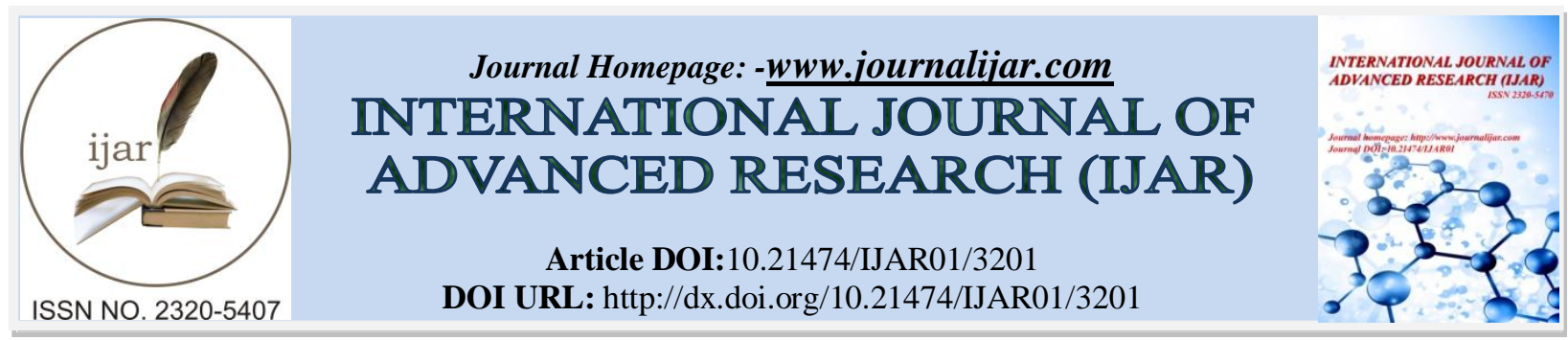

RESEARCH ARTICLE

\title{
CASE REPORT : ORBITAL CELLULITIS IN OLD PATIENT WITH HISTORY OF OCULAR INTERVENTION.
}

\author{
Dr. Fatima al.mulhim ${ }^{1}$, Dr .Anoud al-jamaan ${ }^{1}$, Dr.kauser ${ }^{2}$, Dr.Abdulmohsin al-mulhim ${ }^{3}$, Dr.Muslem Al- \\ besher ${ }^{1}$, Hussain Al-Sinni ${ }^{4}$, Dr. Eman Al kishi ${ }^{1}$ and Dr. Ahmed Al-esmael ${ }^{3}$. \\ 1. Ophthalmology Resident, Aljaber Eye And ENT Hospital, Kingdom Of Saudi Arabia. \\ 2. Ophthalmology Specialist, Aljaber Eye And ENT Hospital, Kingdom Of Saudi Arabia. \\ 3. ER Resident, King Fahd Hospital, Kingdom Of Saudi Arabia. \\ 4. ENT Resident, Aljaber Eye And ENT Hospital, Kingdom Of Saudi Arabia.
}

\section{Manuscript Info}

Manuscript History

Received: 09 December 2016

Final Accepted: 15 January 2017

Published: February 2017

Key words:-

orbital cellulitis , periosteal abscess ,

proptosis , sinusitis associated

ophthalmomlogy

\section{Abstract}

Orbital cellulitis is considered as an ocular emergency which needs urgent evaluation and proper quick action to save the patient vision or even life

The aim of this report is to present a case about orbital cellulitis with the presence of multiple risk factors as sinusitis and ocular intervention and how to approach such patients

\section{Introduction:-}

Orbital cellulitis is an inflammation of the soft tissues of the eye socket posterior the orbital septum,It most commonly occurs when bacterial infection spreads the adjacent paranasal sinuses or other nearby structures such as the face, eyelids or the lacrimal drainage system through the blood streamAlso, It can be caused by a direct trauma to the orbit ,in which traumas may introduce an infectious materials into the orbitother causes were reported such as direct inoculation of the orbit from surgery or Hematogenous spread from bacteremiaOrbital cellulitis is an ocular emergency that not only threatens vision but also can lead to life-threatening complications if left untrated such as cavernous sinus thrombosis, meningitis, and brain abscess.'Careful history taking and physical examination must be done for fast disease recognition and proper management

\section{Case Report:-}

86 years old Female presented to our hospital ER, complaining of right eye Pain , Redness and Swelling of the eyelid for 3 daysIt was gradual in onset, Progressed rapidly within these 3 days with significant decrease of vision .

Regarding her Past history the patient is Known hypertensive for long time on antihypertensive medication with regular follow ups in PHC .

Also ,she reported History of chronic arthritis .

Regarding her Past ocular history, she was diagnosed with nasolacrimal duct obstruction in a previous visit and She underwent right syringing probing in our hospital 5 days prior to the admission . 
The patients Clinical findings were as follows:-

Her Vision in the Right Eye was counting fingers at 2 METERS and the left eye 0.5 both without correction Intraocular pressure was normal in both eyesRight eye examination revealed Upper and lower eyelid tender swelling along with conjunctival congestion and Chemosis.

There was also Non axial proptosis pushing the globe slightly outward and downwards with restriction of ocular movements in all directions .

On slit lamb Anterior segment examination:-

Right Eye:-

There was Grade 1 RAPD

Immature cataract

Fundus: WNL

Left Eye:-

Anterior segment-

Immature cataract

Fundus: within normal level

The patient was admitted to the hospital:-

Investigations:-

CT-scan:-

Axial and coronal CT scan was done with and without contrast done, revealed sub periosteal abcess in the upper medial wall of the orbit originating from the ethmoid sinus which showed signs of sinusitis.

\section{The patient was given the following Treatment:-}

Medical treatment in the form of:

Injection IV Ceftriaxone $1 \mathrm{gm}$ BID

Ciprofloxacin Eyedrops q4 hourly

Chloramphenicol Eye ointment HS

Tablet Brufen $400 \mathrm{mg}$ BID

Tablet Ranitidine 150 mg BID

After the CT scan results came, Surgical treatment started:-

The sub periosteal abscess was drained under general anesthesia through the caruncular approach and the pus was sent for culture and sensitivity.

A swab was also taken.

Postoperative treatment:

Injection IV Ceftriaxone $1 \mathrm{gm}$ BID

Injection Vancomycin 500mg q6hrs

Injection Flagyl 500mg TID

Tab Prednisolone50 mg PO OD

Tab Brufen 400 mg BID

Tab Ranitidine $150 \mathrm{mg}$ BID

Postoperative picture:-

$1^{\mathrm{ST}}$ post operative day

the general condition of the patient improved

Vision: counting fingers 3 meters

The pain was relieved

The lid edema and the chemosis were reduced

The ocular movements had improved 


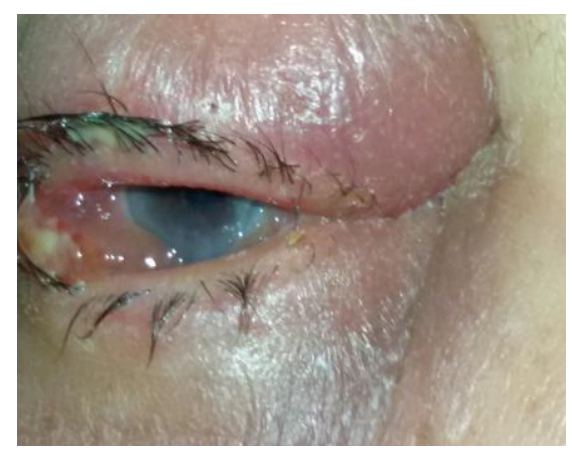

Right eye examination revealed Upper and lower eyelid tender swelling along with conjunctival congestion and Chemosis .

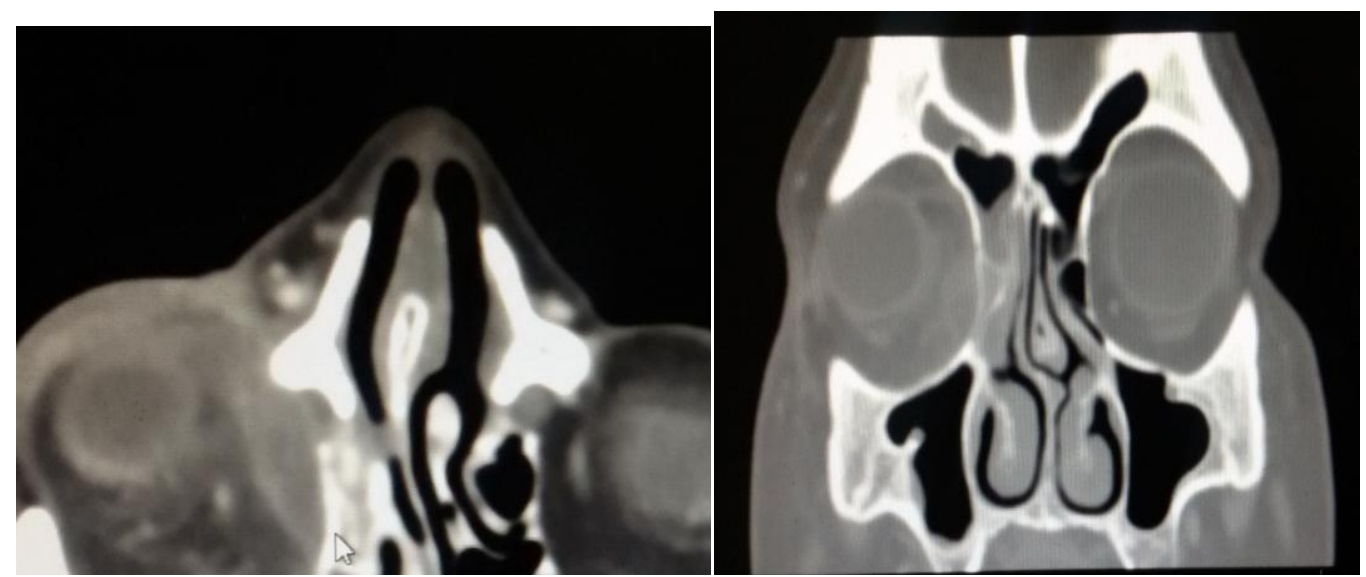

CT Scan without contrast revealed sub-periosteal abscess in the upper medial wall of the orbit originating from the ethmoid sinus which showed signs of sinusitis

\section{Discussion:-}

Displacement of the globe may be due to an increase in the orbital contents. the orbit has rigid bony walls except its anterior wall, such displacement usually manifests predominantly as forward protrusion of the globe which is called proptosis, which is the hallmark of orbital disease.

orbital cellulitis may due to an injury perforating the orbital septum.

The Inflammation process can be noticed within the first 48-72 hours after injury, it may be delayed for several months if retained orbital foreign body.

Some orbital cellulitis cases were reported after surgical procedures, such as orbital decompression, DCR, blepharoplasty, squint surgeries, retinal surgery, and glaucoma surgery.

The diagnosis of orbital cellulitis is usually based on clinical findings with the aid of radiological findings sometimes

Usually, The presence of orbital signs such as proptosis, pain during eye movements, ophthalmoloplegia, optic nerve involvement as well as fever and high WBC count confirm the diagnosis. Age, medical history, the presence of trauma and the mechanism of injury play an important role in determining the causative organism of orbital disease.Staphylococcus species, Streptococcus species and Bacteroides species are the most common organisms detected in adult orbital cellulitis, while unvaccinated children can present with sequelae from Haemophilusinfluenzae. To be noticed, some organisms can be detected In all age groups with specific conditions, foe example, dental abscess with mixed, aggressive aerobic and/or anaerobic infection must be considered if a suggestive history is elicited. Gram-negative rods are likely to be the causative organism recovered in abscesses 
secondary to trauma. Fungal infections, including mucormycosis/zygomycosis and aspergillosis, especially in diabetic or immunocompromised hosts.

\begin{tabular}{|l|l|l|}
\hline Age of the patient & Microbiology results & Surgical management \\
\hline Less than 9 & $\begin{array}{l}\text { No growth or one anaerobic } \\
\text { microorganism Not indicated }\end{array}$ \\
\hline From 9 to 14 & $\begin{array}{l}\text { Mixed aerobic and anaerobic } \\
\text { microorganism }\end{array}$ & Possible \\
\hline More than 14 & Mixed or only anaerobics & Indicated \\
\hline
\end{tabular}

\section{Conclusion:-}

Orbital cellulitis due to Subperiosteal abscess is a well-described infectious process that affects the bones supporting the globe. It can cause rapid clinical deterioration of the bone and intracranial extension. Careful clinical monitoring, serial ophthalmologic examinations and comparative radiologic screening of persons must be done, followed by prompt and appropriate treatment. Thses details are necessary to decrease the risk of complications, such as permanent vision loss, cavernous sinus thrombosis and cerebral abscesses.

Orbital cellulitis is considered as an ocular emergency which needs urgent evaluation and proper quick action to save the patient vion or even life.

\section{References:-}

- John ForresterAndrew Dick Paul McMenamin Fiona Roberts Eric Pearlman, The eye , page 1,2,3,4,

- Richard S. Snell, Michael A. Lemp clinical anatomy of the eye page 70

- Kanski's Clinical Ophthalmology, 6th Edition, A Systematic Approach page 175, 176

- Vaughan, Daniel \& Asbury, Taylor. General Ophthalmology. Los Altos, CA. Lange Medical Publications, 1958. (15 ${ }^{\text {th }}$ edition 1999 , with Riordan-Eva, P. Appleton, Lange) chapter 13 , the orbit

- Renu Jogi, basic opthalmomlogy, the orbit page 439

- British Journal of Ophthalmology, 1989, 73, 205-208 Orbital cellulitis: review of 23 cases from Saudi Arabia

- American Academy of Ophthalmology. Basic and Clinical Science Course. San Francisco, CA A.A.O. 19402000 eyewiki. Orbital cellulitis 